\title{
Investigation of Multicritical Phenomena in ANNNI Model by Monte Carlo Methods
}

\author{
A. K. Murtazaev ${ }^{1,2}$ and J. G. Ibaev ${ }^{1}$ \\ ${ }^{1}$ Institute of Physics, Daghestan Scientific Center of RAS, Yaragskogo Street 94, Makhachkala 367003, Russia \\ ${ }^{2}$ Daghestan State University, Gadzhieva Street 43a, Makhachkala 367025, Russia \\ Correspondence should be addressed to J. G. Ibaev, ibaev77@mail.ru
}

Received 20 July 2011; Accepted 16 December 2011

Academic Editor: Vladimir Shavrov

Copyright (c) 2012 A. K. Murtazaev and J. G. Ibaev. This is an open access article distributed under the Creative Commons Attribution License, which permits unrestricted use, distribution, and reproduction in any medium, provided the original work is properly cited.

\begin{abstract}
The anisotropic Ising model with competing interactions is investigated in wide temperature range and $\left|J_{1} / J\right|$ parameters by means of Monte Carlo methods. Static critical exponents of the magnetization, susceptibility, heat capacity, and correlation radius are calculated in the neighborhood of Lifshitz point. According to obtained results, a phase diagram is plotted, the coordinates of Lifshitz point are defined, and a character of multicritical behavior of the system is detected.
\end{abstract}

\section{Introduction}

Apart from problems of critical phenomena, more complex phenomena observed near the diagram special points of system states, where the lines of different order phase transitions cross, are heavily emphasized [1]. These phenomena, conditionally named "multicritical," are possible in those systems, where a symmetry and interparticle interaction assumes several types of ordering. Similar phenomena are observed in a mixture of gases, liquids, ferroelectrics, magnetics, and so forth.

An experimental investigation of the multicritical phenomena is more complex than theoretical. This complexity has to do with the fact that in the neighborhood of multicritical point, a region with different strongly developed fluctuations broadens, and the crossovers are probable [2].

A theoretical study of multicritical phenomena results in complex nonlinear differential equations. Exact analytical solution of these equations also entails great difficulties [3]. In this connection the methods of computational physics (Monte Carlo method (MC)) and molecular dynamics (MD) became basic techniques to study those equations at present. In practice, for description of such complex systems, different models are used. The simplest and effective one among them is anisotropic Ising model with competing interactions with second nearest neighbors (ANNNI model,
Figure 1). We plot a phase diagram for this model and study a character of the critical behavior in Lifshitz point using the standard method of Monte Carlo.

\section{Model}

ANNNI model appeared in the static physics in the second half of XX century for explanation of the spiral magnetic order in heavy rear-earth metals [4]. Its Hamiltonian in perfect cubic lattice has the following form:

$$
H_{\mathrm{ANNNI}}=-J \sum_{i, j} s_{i} s_{j}+J_{1} \sum_{i} s_{i} s_{i+1}
$$

where $s_{i}= \pm 1, J$ denotes the parameter of exchange interaction of neighboring spin pairs, and $J_{1}>0$ is the parameter of antiferromagnetic exchange of neighbors following the nearest neighbor along $Z$ axis.

A topology of $T-\left|J_{1} / J\right|$ phase diagram includes three regions for three-dimensional case (3D), which are crossing in multicritical Lifshitz point [5]. The system is paramagnetic at high temperatures and is ferromagnetic at low $T$ and small $\left|J_{1} / J\right|$; when $\left|J_{1} / J\right|$ has sufficiently large values, the modulated phases are generated (Figure 2).

According to the literature data when temperature decreasing ANNNI model undergoes the second-order phase 


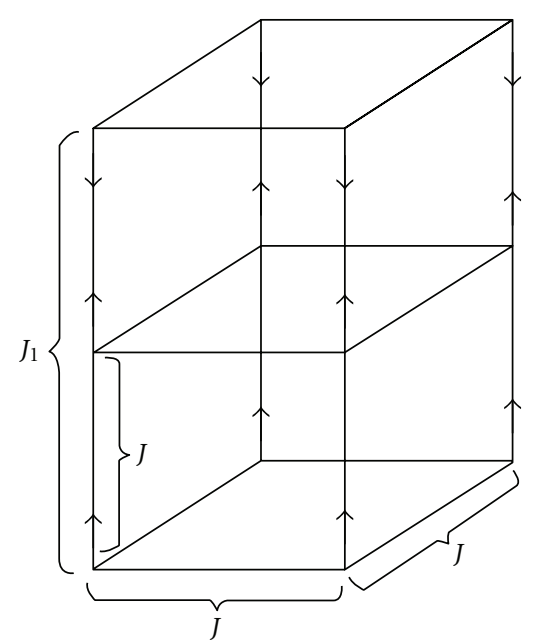

FIgURE 1: Anisotropic model with competing interactions.

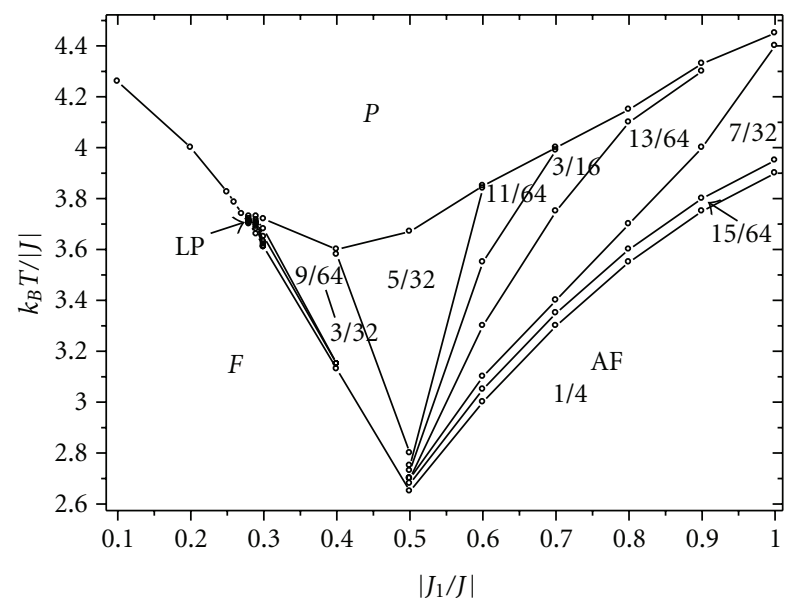

FIGURE 2: Phase diagram of anisotropic Ising model with competing interactions (by the data of [6]).

transition from paramagnetic state to the nearest ordered state, and a transition "ferromagnetic-modulated state" is the first-order transition [7].

For description of a phase behavior of studied model, we used approximated theoretical methods including high- and low-temperature expansions [8], the mean-field theory [9], and other theoretical approximations [10].

The Monte Carlo modeling [11] mainly was carried out in a region of "ferromagnetic-paramagnetic" transition up to the Lifshitz point [12], but a precise determination of the transition in the point and its nearest neighborhood remains unsolved.

According $t$ data in $[6,12]$, a critical behavior of the systems with modulated structures at small values of $\left|J_{1} / J\right|$ is described by the Ising universality class of the critical behavior. When increasing $\left|J_{1} / J\right|$ a crossover to the critical behavior typical for the Lifshitz point occurs and from it to XY-critical behavior.

\section{Method of Investigation}

The cubic systems with periodic boundary conditions and sizes $L \times L \times L, L=12 \div 64$ were used for investigation of multicritical phenomena in the vicinity of the Lifshitz point. Spin number in studied systems was $N_{\text {eff }}=512 \div$ 262144. Markov chains of $\tau=1000 \tau_{0}$ length $\left(\tau_{0}=10^{4}\right.$ MC-step/spin is a length of nonequilibrium part) were generated on the computer. The thermodynamic parameters of the system were calculated by averaging along this chain. Moreover, the averaging was carried out by 1000 different initial configurations. An error of obtained results does not exceed the sizes of symbols used in Figures.

The temperature dependences of the heat capacity and susceptibility were derived by means of expressions [9]

$$
\begin{gathered}
C=\left(N K^{2}\right)\left(\left\langle U^{2}\right\rangle-\langle U\rangle^{2}\right), \\
\chi=(N K)\left(\left\langle m^{2}\right\rangle-\langle m\rangle^{2}\right),
\end{gathered}
$$

where $K=|J| / k_{B} T$; $U$ denotes the intrinsic energy; $m$ indicates the magnetization.

The critical temperature was estimated by the Binder cumulant method. According to the finite-size scaling theory (FSS) [13], the cumulants

$$
U_{L}=1-\frac{\left\langle m^{4}\right\rangle}{3\left\langle m^{2}\right\rangle^{2}},
$$

for systems with different sizes $L$, cross in a critical point $T_{c}$. Plotting the $U_{L}$ temperature dependences for systems with different sizes $L$, one can estimate the critical temperatures of systems. Critical temperatures values calculated by this method for different values of $\left|J_{1} / J\right|$ are presented in Table 1 .

The critical parameters of the magnetization, susceptibility, and correlation radius were estimated from a ratio of FSS theory [14].

For calculation of the magnetic properties of modulated region, we used a value

$$
M_{Z}=\frac{1}{L^{2}} \sum_{x, y}^{L} S_{x, y, z}
$$

being the averaged magnetization of the layer, which is perpendicular to $Z$ axis.

The values of a wave number and a character of amplitude and phase modulation were detected by means of mathematical apparatus of the spectral analysis based on the Fourier transformations [15].

\section{Results and Discussion}

Using Fourier transformations, we succeeded, with sufficiently high accuracy, to calculate the existence domains of modulated phases with different values of the wave length and estimate a stability of the modulated phases. If on their Fourier transforms, there are observed clearly defined peaks at a value of $k$, one can consider with confidence that the structure is stable in the given temperature range and ratio of interaction constants. The appearance of side 
TABLE 1: Critical parameters of the ANNNI model.

\begin{tabular}{llllllllll}
\hline$\left|J_{1} / J\right|$ & $T_{c}$ & $\alpha / \nu$ & $\beta / \nu$ & $\gamma / \nu$ & $1 / \nu$ & $\alpha$ & $\beta$ & $\gamma$ & $\nu$ \\
\hline 0,271 & $3,736(1)$ & 0,352 & 0,431 & 2,499 & 1,799 & $0,196(4)$ & $0,240(4)$ & $1,389(6)$ & $0,556(6)$ \\
0,272 & 3,732 & 0,379 & 0,429 & 2,719 & 1,903 & 0,199 & 0,225 & 1,427 & 0,525 \\
0,273 & 3,728 & 0,420 & 0,422 & 2,880 & 2,055 & 0,203 & 0,206 & 1,403 & 0,487 \\
0,274 & 3,724 & 0,460 & 0,421 & 3,053 & 2,183 & 0,211 & 0,193 & 1,398 & 0,458 \\
0,275 & 3,726 & 0,449 & 0,422 & 3,002 & 2,125 & 0,211 & 0,199 & 1,414 & 0,471 \\
0,276 & 3,727 & 0,461 & 0,422 & 3,117 & 2,225 & 0,207 & 0,189 & 1,400 & 0,449 \\
\hline Ising & 4,511 & & & & & 0,113 & 0,3258 & 1,239 & 0,63 \\
\hline
\end{tabular}

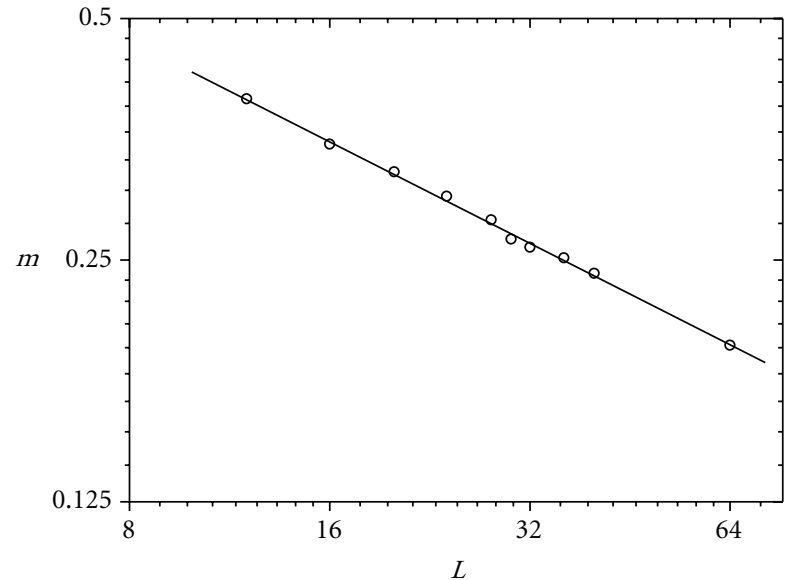

FIGURE 3: Log-log scale dependence of the magnetization.

peaks above and below the main maximum on the Fourier transformations indicates that the system is in a region of transition from one modulated structure to another. A transition of the system from a modulated phase into the paramagnetic phase can be detected by a sharp increase of peaks on the Fourier transformations, what confirms the continuity of its spectrum.

Generalizing the obtained results, we plotted a $\left|J_{1} / J\right|$, $k_{B} T /|J|$ phase diagram (Figure 2). The second-order phase transition dependences were calculated by the Binder cumulants method, and lines of transitions in the modulated region were derived by the results of Fourier analysis. A temperature in which uneven change in wave number of modulated structures occurs was taken as a transition temperature.

As it is evident from plotted phase diagram, the multicritical Lifshitz point is revealed in the intervals $0,27<\left|J_{1} / J\right|<$ 0,28 and 3,74<T<3,72. Therefore, all experimental data of the critical parameters presented in the work belong to the given range of parameter change of $\left|J_{1} / J\right|$ and $T$.

To determine the critical exponents, we plotted the loglog scale curves for the dependences of thermodynamic parameters on system linear sizes at $T=T_{c}$. Figures 3 and 4 present the characteristic dependences of magnetization and parameters of $V_{i}$ at $i=1,2,3$ on linear sizes at $\left|J_{1} / J\right|=0,273$. As it is clear from Figures, all points on curves lie down a straight line within an error. The inclination of line detects

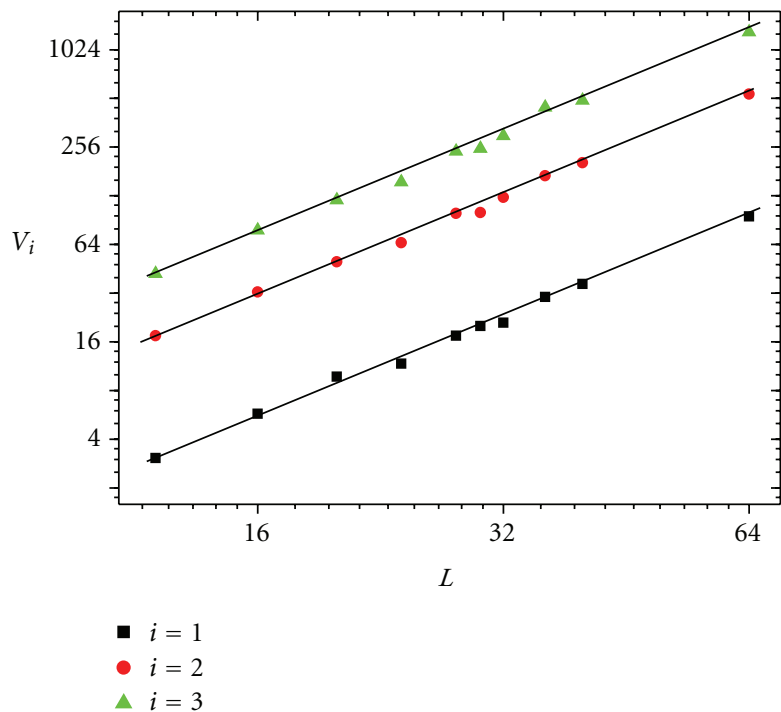

Figure 4: Log-log scale dependence of $V_{i}$ on the system linear sizes.

the values of $\beta / \nu$ in Figure 3 and of $1 / \nu$ in Figure 4 . By the same dependences the values of $\alpha / \nu$ and $\gamma / \nu$ were defined. The obtained values of the critical exponents are shown in Table 1.

According to data in the Table 1, a character of change of critical parameters up to $\left|J_{1} / J\right|<0,274$ is the same as in work [6]. Such a behavior is connected with the crossover from the critical behavior to the multicritical and inversely. Obviously, behavior of critical parameters at $\left|J_{1} / J\right|>0,274$ is due to the fact that our system starts to aberrate from multicritical behavior, and, consequently, the Lifshitz point is localized in range of $\left|J_{1} / J\right|=0,274$ and $T=3,724$.

It should be observed that, in this work, unlike previous work [6], the critical exponents of correlation radius are estimated. A change of $v$ values with increase in $\left|J_{1} / J\right|$, probably, has to do with the region that with strongly developed fluctuations widens at approximation to the multicritical point.

In conclusion, let us note that a critical behavior of majority of crystals in the vicinity of phase transitions differs from being predicted for corresponding universality classes. A reason for such behavior is various anisotropic and competitive interactions existing in real systems. A consideration of all possible kinds of anisotropy and interactions can 
lead to the multicritical phenomena, which appear on the phase diagrams as intersection point of phase transitions curves (a typical example-the Lifshitz point). Therefore, the investigation of similar phenomena is also important condition for studying the critical phenomena. A design of new methods of investigation and algorithms of modeling for estimation of multicritical phenomena can favour the study not only of the Lifshitz point neighborhoods but amplify the application of Monte Carlo methods for research of more complex systems.

\section{Acknowledgment}

Support by RFBR (no. 09-02-96506), and PHCP (no. П554 and no. 02.740.11.03.97) is acknowledged.

\section{References}

[1] P. A. Fleury, "Phase transitions, critical phenomena, and instabilities," Science, vol. 211, no. 4478, pp. 125-131, 1981.

[2] M. A. Anisimov, E. E. Gorodetskii, and V. M. Zaprudskii, "Phase transitions with coupled order parameters," Soviet physics, Uspekhi, vol. 24, pp. 57-75, 1981.

[3] Yu. A. Izumov and V. M. Siromyatnikov, Phase Transitions and Crystal Symmetry, Springer, 1984.

[4] W. Selke, "The ANNNI model-theoretical analysis and experimental application," Physics Reports, vol. 170, no. 4, pp. 213-264, 1988.

[5] R. M. Hornreich, M. Luban, and S. Shtrikman, "Critical behavior at the onset of $\mathrm{k} \rightarrow$-space instability on the $\lambda$ line," Physical Review Letters, vol. 35, no. 25, pp. 1678-1681, 1975.

[6] A. K. Murtazaev and Z. G. Ibaev, "Critical properties of the anisotropic ising model with competing interactions," Journal of Experimental and Theoretical Physics, vol. 113, no. 1, pp. 106-112, 2011.

[7] A. Gendiar and T. Nishino, "Phase diagram of the threedimensional axial next-nearest-neighbor Ising model," Physical Review B, vol. 71, no. 2, Article ID 024404, 7 pages, 2005.

[8] S. Redner and H. E. Stanley, "Helical order and its onset at the Lifshitz point," Physical Review B, vol. 16, no. 11, pp. 49014906, 1977.

[9] P. Peczak, A. M. Ferrenberg, and D. P. Landau, "Highaccuracy Monte Carlo study of the three-dimensional classical Heisenberg ferromagnet," Physical Review B, vol. 43, no. 7, pp. 6087-6093, 1991.

[10] P. Bak and J. Von Boehm, "Ising model with solitons, phasons, and 'the devil's staircase," Physical Review B, vol. 21, no. 11, pp. 5297-5308, 1980.

[11] K. Kaski and W. Selke, "Monte Carlo coarse graining for the three-dimensional axial next-nearest-neighbor Ising model," Physical Review B, vol. 31, no. 5, pp. 3128-3130, 1985.

[12] M. Pleimling and M. Henkel, "Anisotropic scaling and generalized conformal invariance at Lifshitz points," Physical Review Letters, vol. 87, no. 12, Article ID 125702, 4 pages, 2001.

[13] K. Binder, "Critical properties from Monte Carlo Coarse graining and renormalization," Physical Review Letters, vol. 47, no. 9, pp. 693-696, 1981.

[14] A. K. Murtazaev, I. K. Kamilov, and K. K. Aliev, "Finite-size scaling and critical exponents of the real antiferromagnetic model," Journal of Magnetism and Magnetic Materials, vol. 204, no. 1, pp. $151-158,1999$.
[15] R. J. Elliott, "Phenomenological discussion of magnetic ordering in the heavy rare-earth metals," Physical Review, vol. 124, no. 2, pp. 346-353, 1961. 

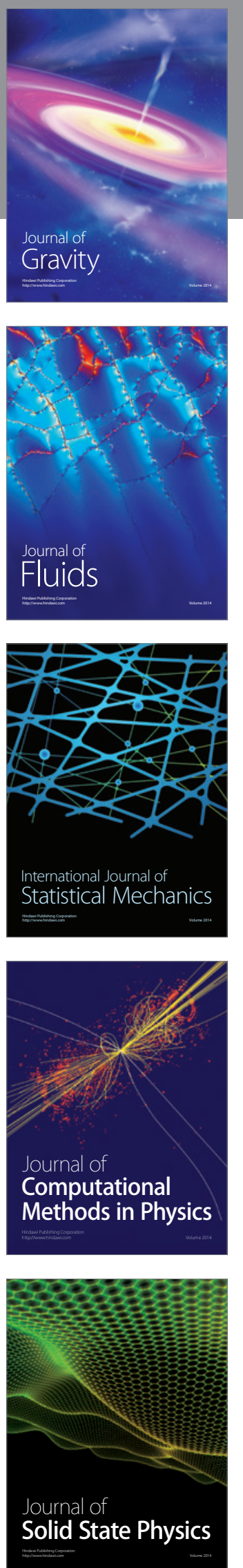

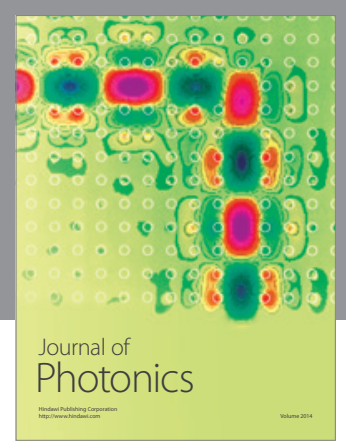

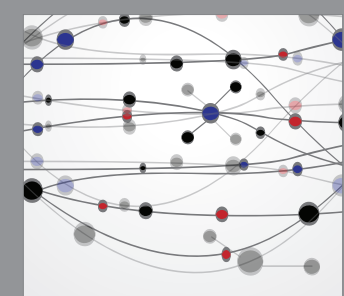

The Scientific World Journal
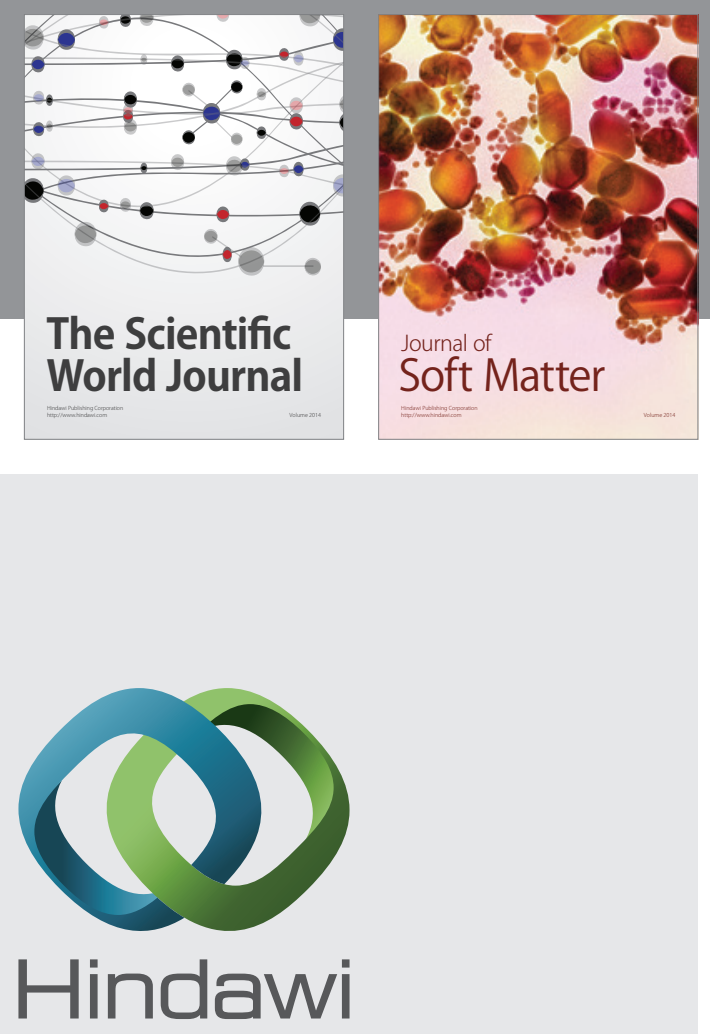

Submit your manuscripts at

http://www.hindawi.com
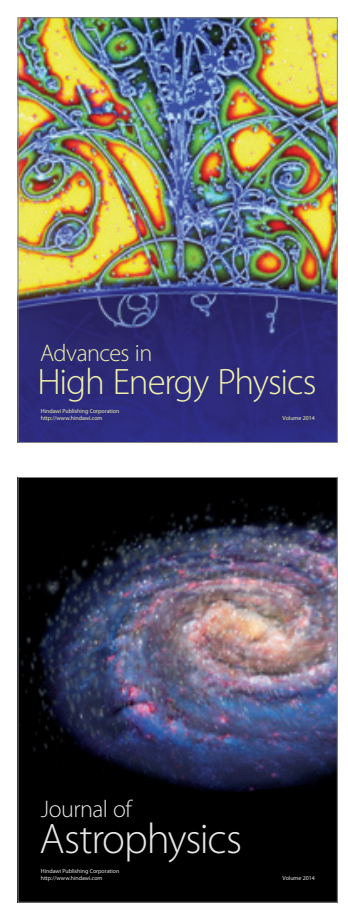
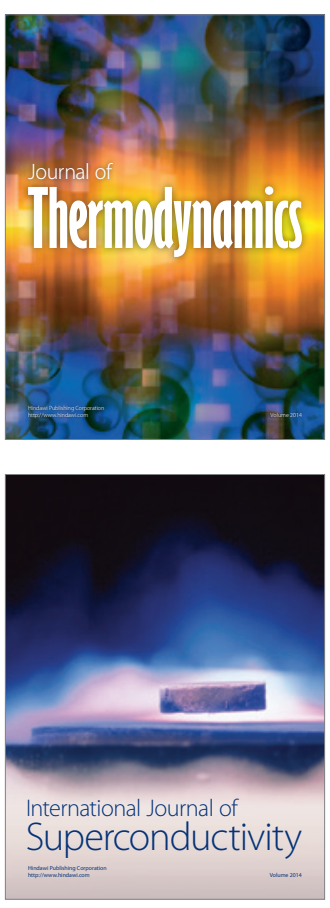
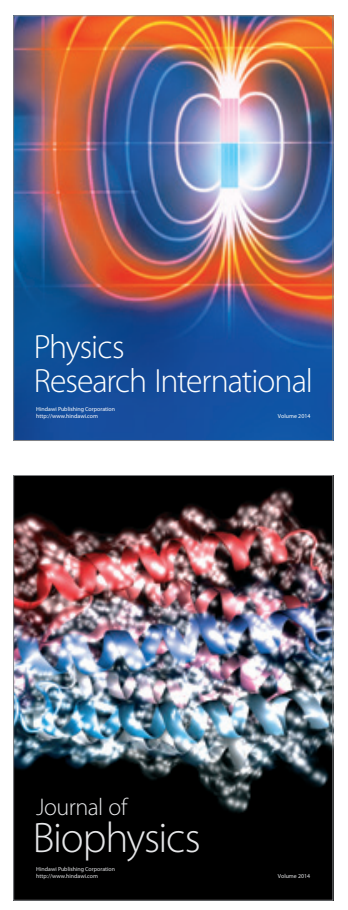
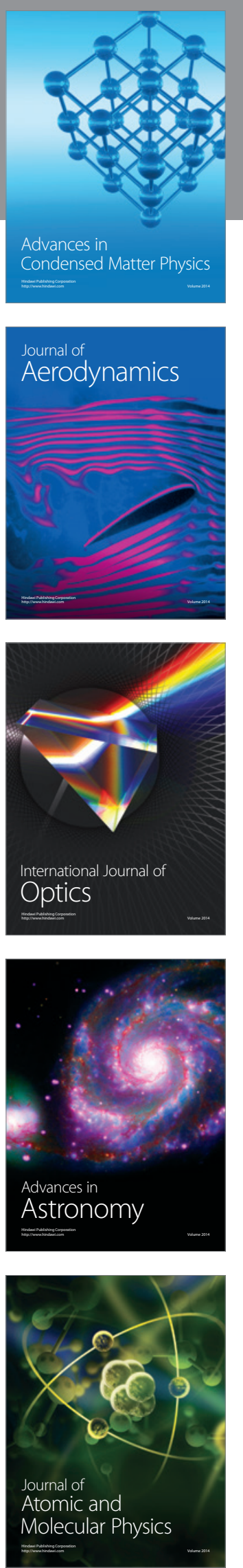